\title{
OPTIMAL PATH PLANNING FOR BACKHOE BASED ON EXCAVATION ENVIRONMENT
}

\author{
Hyongju Park, Jin-Ok Shin, Sanghak Lee and Daehie Hong \\ Dep. of Mechanical Engineering, \\ Korea University, \\ 1-5 Anamdong, Sungbukgu, \\ Seoul, 136-701, Korea \\ \{hyongju, jinokshin, sanghak, dhhong\}@korea.ac.kr
}

\begin{abstract}
The paper focuses on the establishment of optimized bucket path planning using numerical solution method, for a force-reflecting backhoe which is affected by excavation environment including potential obstacles. The developed path planning method can be used for precise bucket control and more importantly for obstacle avoidance which is directly related to safety issues. An optimal path planning model to minimize energy and to move manipulator arms along a specified path subjected to angular constraints was first established. Simultaneously, path modification due to unexpected obstacles was considered. A Newton-Raphson iteration method was used to acquire angular information of boom, stick, and bucket to solve inverse kinematics. The model presented in this paper was intended to be implemented in further study of force and vibration feedback control of tele-operated excavator using haptic devices.
\end{abstract}

\section{KEYWORDS}

Obstacle Avoidance, Joint Constraint, Jacobian Pseudo-Inverse, Path Planning

\section{INTRODUCTION}

Excavation of large area by human operator not only takes a lot of time, but also can lead to high labor cost. In addition, excavation environment is recognized as one of the most hazardous construction environment. Therefore, automation of excavation is essential to resolve both time and economic inefficiency and potential risks inherited in excavation environment. Many efforts have been made on development of automated and intelligent excavator $[1,2,3]$. One of the requisites for the accomplishment of fully automated excavation environment is establishment of optimal path planning of excavator. Optimum path algorithm must be constructed in a way of energy efficient and also obstacle avoidance ready. To attain both goals, numerical method was used for deriving solutions of inverse kinematic problem.

For a redundant manipulator there are infinite numbers of solution exist. Backhoe can be classified as redundant manipulator for the reason that it has 4 DOFs including swing, boom, stick, and bucket rotational commands, while there are three independent variables to describe the motion of the manipulator. As the method for acquiring solution, Newton-Raphson iteration was used to find minimum norm solutions for commanded end-effector motion. Minimum norm solution is not always the best choice when there is a potential obstacle inside the manipulator workspace or divergences of solution occurs due to near singularity configuration of links. Therefore, it is important to achieve the second goal of obstacle avoidance and the third goal of moving the links within joint angle limits while 
satisfying the first goal of moving end-effector to desired position and orientation. Successful task is therefore defined as achieving all of these goals simultaneously with smooth conversion between them. Setting boundary of joint angle limit prevents manipulator from undergoing serious damage of equipments due to robust motion. For discriminating proper joint angle limits within the joint null space, a scheme to obtain directionality of joint angles with respect to end-effector motion was established. Simulations were conducted to verify the joint directionality identification scheme. Assignment of link and joint variables, joint axes was built upon Denavit-Hartenberg notation [4].

\section{THEORY}

The end-effector position and orientation vector for manipulator can be expressed by $m$ by 1 matrix, where $m$ equals 6 in general case of redundant manipulator in Cartesian space. Jointspace vector can be represented as $n$ by 1 matrix where $n$ corresponds to degrees of freedom the manipulator has. An equation which relates Cartesian vectors $x_{e}(t) \in R^{m}$ to joint space vectors is $\theta(t) \in R^{n}$ as the following.

$x_{e}(t)=f(\theta(t))$

By taking time derivatives of each parameter, linear correspondence between Cartesian space vector and joint space vector can be achieved.

$\dot{x}_{e}(t)=J_{e} \dot{\theta}(t)$

where $J_{e}$ is $m$ by $n$ end-effector Jacobian matrix. The general solution to the equation (2) is given by Rao et al. [5],

$$
\dot{\theta}(t)=J_{e}^{+} \dot{x}_{e}(t)+\left(I-J_{e}^{+} J_{e}\right) z
$$

where $J_{e}^{+}$denotes the Moore-Penrose pseudoinverse given by $J_{e}^{+}=J_{e}^{T}\left(J_{e} J_{e}^{T}\right)^{-1}, J_{e}$ matrix is $m<n$ according to (2), which means manipulator is redundant. $\left(I-J_{e}^{+} J_{e}\right)$ is projection operator which chooses vectors from $z$ and send them to null space to satisfy (2). $z$ is an arbitrary vector in joint space, which can be altered properly as far as it is located in the space of homogeneous solution. Yoshikawa [6] has implemented obstacle avoidance scheme for selecting vector $z$, which mainly describes the relationship between $z$ and specified velocity vector moving to collision-free or safe place. The minimal obstacle distance was obtained earlier by Hanafusa et al. [7], and vector $z$ was given by

$$
z=\left[J_{o}\left(I-J_{e}^{+} J_{e}\right)\right]^{+}\left(\dot{x}_{o}-J_{o}^{+} J_{e} \dot{x}_{e}\right)
$$

where $J_{o}$ is obstacle Jacobian which is formulated from specified velocity vector acting to critical point on link. The location of critical points were described in paper by Zhang et al. [8]. Maciejewski et al. [9] put multiple obstacle avoidance scheme upon work by Hanafusa et al. [7]. By substituting (4) into (3),

$\dot{\theta}(t)=J_{e}^{+} \dot{x}_{e}(t)+\left(I-J_{e}^{+} J_{e}\right)\left[J_{o}\left(I-J_{e}^{+} J_{e}\right)\right]^{+}\left(\dot{x}_{o}-J_{o}^{+} J_{e} \dot{x}_{e}\right)(5)$

\section{IMPLEMENTATION}

While implementing the theory, it was found that the directionality of distal point of specific links or joints can be recognized and selected by substituting $J_{o}$ with $J_{k}$ where $k$ is the joint number $[k=2,3, \ldots]$ in control. Firstly, two

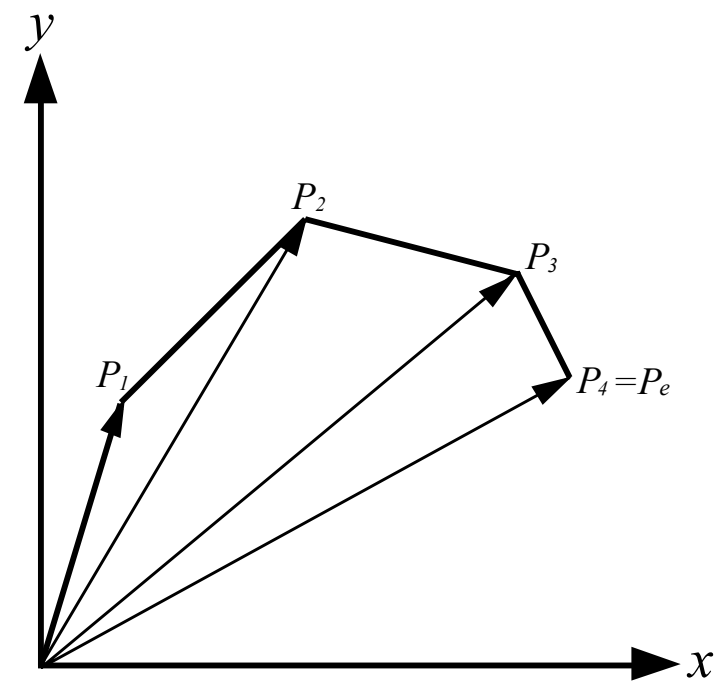

Figure 1 Definition of $P_{k}$ for 4 Link Manipulator 
dimensional redundant manipulator having four links was considered. Components of $J_{k}$ matrix was given by,

$$
J_{k}=\left[\begin{array}{llll}
\frac{\partial P_{k, x}}{\partial \theta_{1}} & \frac{\partial P_{k, x}}{\partial \theta_{2}} & \frac{\partial P_{k, x}}{\partial \theta_{3}} & \frac{\partial P_{k, x}}{\partial \theta_{4}} \\
\frac{\partial P_{k, y}}{\partial \theta_{1}} & \frac{\partial P_{k, y}}{\partial \theta_{2}} & \frac{\partial P_{k, y}}{\partial \theta_{3}} & \frac{\partial P_{k, y}}{\partial \theta_{4}}
\end{array}\right]
$$

It can be seen from Fig. 1 that $P_{k}=\left[P_{k, x}, P_{k, y}\right]^{T}$ is vector of joint number $k$ from its origin. $J_{k}$ cannot be directly obtained by differentiating $P_{k}$ with respect to $\theta_{l}$; where $l=1,2,3,4$ for 4 link manipulator. The reason is for the case $k<l$, when deriving partial derivative of $P_{k}$ with respect to $l$-th variable of $\theta$, every elements in $l$-th column of becomes zero, which makes $J_{k}$ meaningless. There are two ways to express coordinates of $P_{k}$ in Cartesian space. That is to either use direct kinematics and represent the position vector with respect to origin or inverse kinematics and represent the position from end-effector.

$$
P_{k}=\left\{\begin{array}{cll}
P_{k}, & \text { if } & k \geq l \\
P_{e}+P_{k}^{e}, & \text { if } & k<l
\end{array}\right.
$$

As shown in Fig. 2, $P_{e}$ is end effector point and $P_{k}^{e}$ is vector from $P_{e}$ to $P_{k}$. Both vectors are pointing at a same spot in terms of Cartesian coordinate but having different orientation. This orientation difference is a key thing for valid formulation of. $J_{k}$. That is, when taking partial derivatives of $P_{k}$ from (7) with respect to $\theta_{l}$ there were no more null elements in the $l$-th column of Jacobian $J_{k}$. Instead of Maciejewski's implementation [9] of obstacle avoidance scheme upon Yoshikawa's work [6], end point velocity of links can be substituted into (5) with gain added to each terms.

Jacobian relating $\dot{x}_{k}(t)$ and $\dot{\theta}(t)$ is given by

$$
\dot{x}_{k}(t)=\lim _{t \rightarrow 0}\left[d P_{k} / d t\right]
$$

where $J_{k}$ is represented by (6), and

$$
\dot{x}_{k}(t)=J_{k} \dot{\theta}(t)
$$

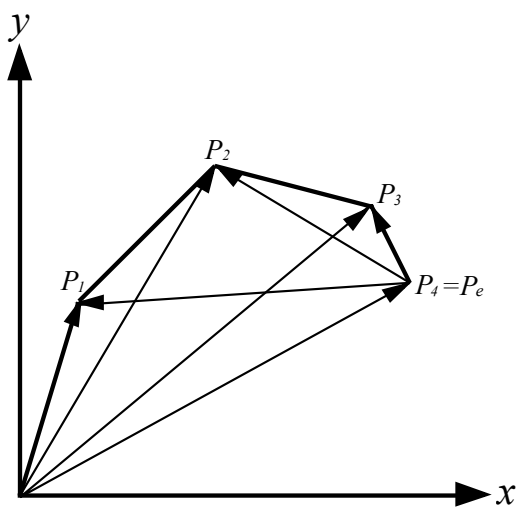

Figure 2 Two Vectors Pointing at a Same Point

Let minimum norm solution satisfying goal for obstacle avoidance, in the second term in (5) as $H_{o}$. Then,

$H_{o}=\left(I-J_{e}^{+} J_{e}\right)\left[J_{o}\left(I-J_{e}^{+} J_{e}\right)\right]^{+}\left(\dot{x}_{o}-J_{o}^{+} J_{e} \dot{x}_{e}\right)(10)$

Likewise, $H_{k}$; the minimum norm solution satisfying goal for each link, is given by

$H_{k}=\left(I-J_{e}^{+} J_{e}\right)\left[J_{k}\left(I-J_{e}^{+} J_{e}\right)\right]^{+}\left(\dot{x}_{k}-J_{k}^{+} J_{e} \dot{x}_{e}\right)$

Modified general solution yields,

$$
\dot{\theta}(t)=s_{e} J_{e}^{+} \dot{x}_{e}(t)+s_{o} H_{o}+\sum_{k=1}^{n} s_{k} H_{k}
$$

where, $s$ subscript denotes respective gain for each term depending on link configuration, which is going to be dealt with later on. $\dot{x}_{k}(t)$ should be assigned in an adequate manner so as to satisfy goal of constraining each joints regardless of end effector motion. To achieve the goal, relation between directionality of each joints and end effector motion should be defined first. Fig. 3 shows an example when $k=1$, that is where rotation of link 1 is concerned. New coordinate frame $x_{k e}-y_{k e}$ was defined. $x_{k e}$ is an axis passing through both points $P_{k}$ and $P_{e}$, and $y_{k e}$ axis is an axis perpendicular to $x_{k e}$ axis and passing through point $P_{e}$ which is current end effector point. 


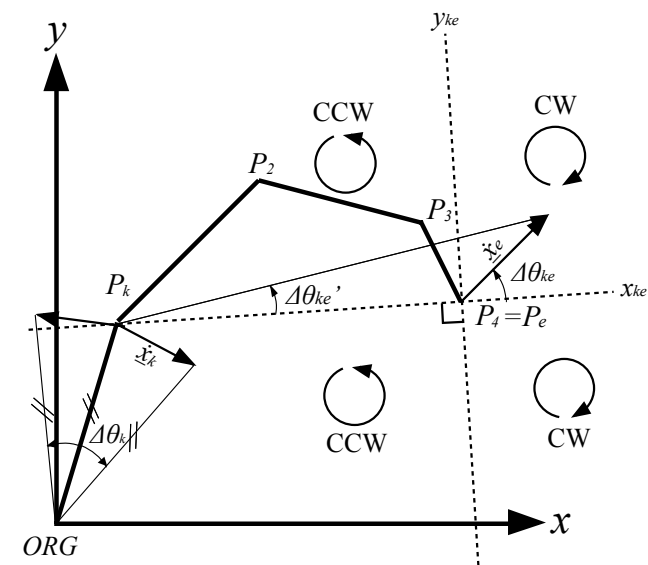

Figure 3 Estimated Rotation of Link 1 w.r.t. ORG Due to End-Effector Movement When $k=1$

If $\Delta \theta_{k e}$, which is an angle made by $\overrightarrow{\dot{x}}_{e}$ and $x_{k e}$ axis, is at $1^{\text {st }}$ or $4^{\text {th }}$ quadrant, link $k$ is rotated clockwise with respect to joint axis $k-1$ from current position. Otherwise when $\Delta \theta_{k e}$ is at $2^{\text {nd }}$ or $3^{\text {rd }}$ quadrant, link is rotated counter-clockwise as you can see in Fig. 3. For clarity, the following equation was constructed.

$\dot{x}_{k}(t)=-D \frac{1}{M} \operatorname{sgn}\left[P_{e}^{k} \square \dot{x}_{e}\right] \Delta \theta_{k e} P_{k}^{k-1}+\dot{x}_{k-1}(t)$

where $D$ is the directional operator and $M$ is the Joint angle magnification factor, and

$$
\Delta \theta_{k e}{ }^{\prime}=a \tan 2\left[\frac{\left\|P_{e}^{k} \times\left[\dot{x}_{e}+P_{e}^{k}\right]\right\|}{\left\|P_{e}^{k}\right\|\left\|\dot{x}_{e}+P_{e}^{k}\right\|}, \frac{P_{e}^{k}\left[\dot{x}_{e}+P_{e}^{k}\right]}{\left\|P_{e}^{k}\right\|\left\|\dot{x}_{e}+P_{e}^{k}\right\|}\right]
$$

The joint directional operator $D$ takes either +1 or -1 depending on user objectives, as is stated on the following.

$D=\left\{\begin{array}{cc}+1, & \begin{array}{c}\text { if want to add robustness and } \\ \text { directionality to the motion of link k }\end{array} \\ -1, & \text { if want to hold the current } \\ \text { position of link k }\end{array}\right.$

Equation (13) is based on the assumption that

$\left\|\dot{x}_{e}\right\|=\left\|\dot{x}_{k}\right\|$.

To satisfy the condition (16), variable $M$ was used. As can be seen in Fig. 4, $M$ was constructed from

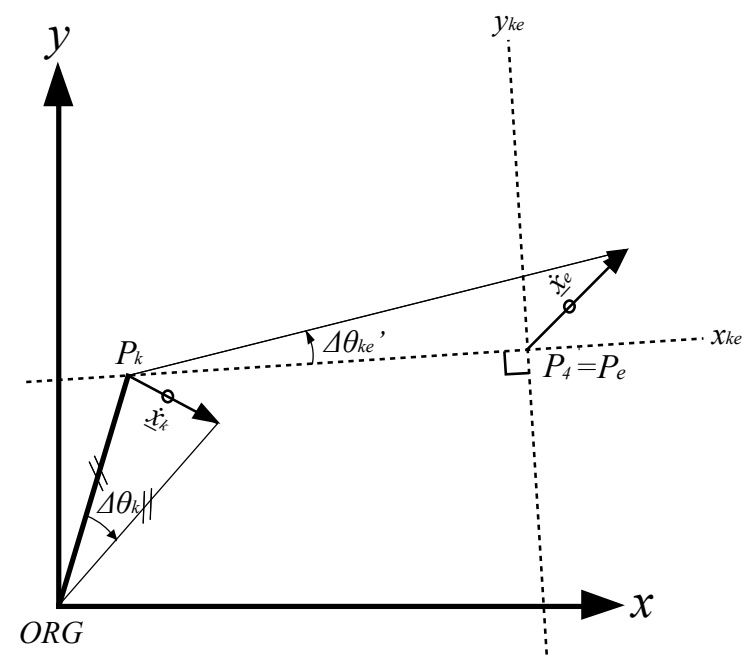

Figure 4 Relation Between Angle Variations, $\Delta \theta_{k e}{ }^{\prime}$ and $\Delta \theta_{k}$ for the Definition of $\boldsymbol{M}$ when $\boldsymbol{k}=\mathbf{1}$

simple relationship between angular velocity and linear velocity. For small angle variation of $\Delta \theta_{k e}{ }^{\prime}$.

$$
\begin{aligned}
& \lim _{\Delta \theta_{k e} \rightarrow 0}\left\|\dot{x}_{e}+P_{e}^{k}\right\|=\left\|P_{e}^{k}\right\| \\
& M \cong \frac{\left\|P_{e}^{k}\right\|}{\left\|P_{k}^{k-1}\right\|}
\end{aligned}
$$

\section{RESULTS}

Before applying joint directionality scheme to backhoe model, simulation for 4-link manipulator was conducted. For clarity, simple point to point command with single point obstacle was considered. One hundred times of iteration was applied to verify stability of the system. Respective gains for each term which is denoted as $s$ subscript was changed properly for respective purpose of tasks. Firstly, by setting every gain terms to zero except for end effector gain, manipulator arm ignored the point obstacle while moving to commanded end effector point.

Results for second case which is to verify the effectiveness of $s_{o}$ (gain for obstacle avoidance term) for the system can be seen in Fig. 5. As it can be seen, end effector has failed to reach the commanded point. Even by adding more numbers of iteration, end effector could not turn around the half circle of obstacle avoidance point completely. 
(a) Trace of each joint for single point to point command
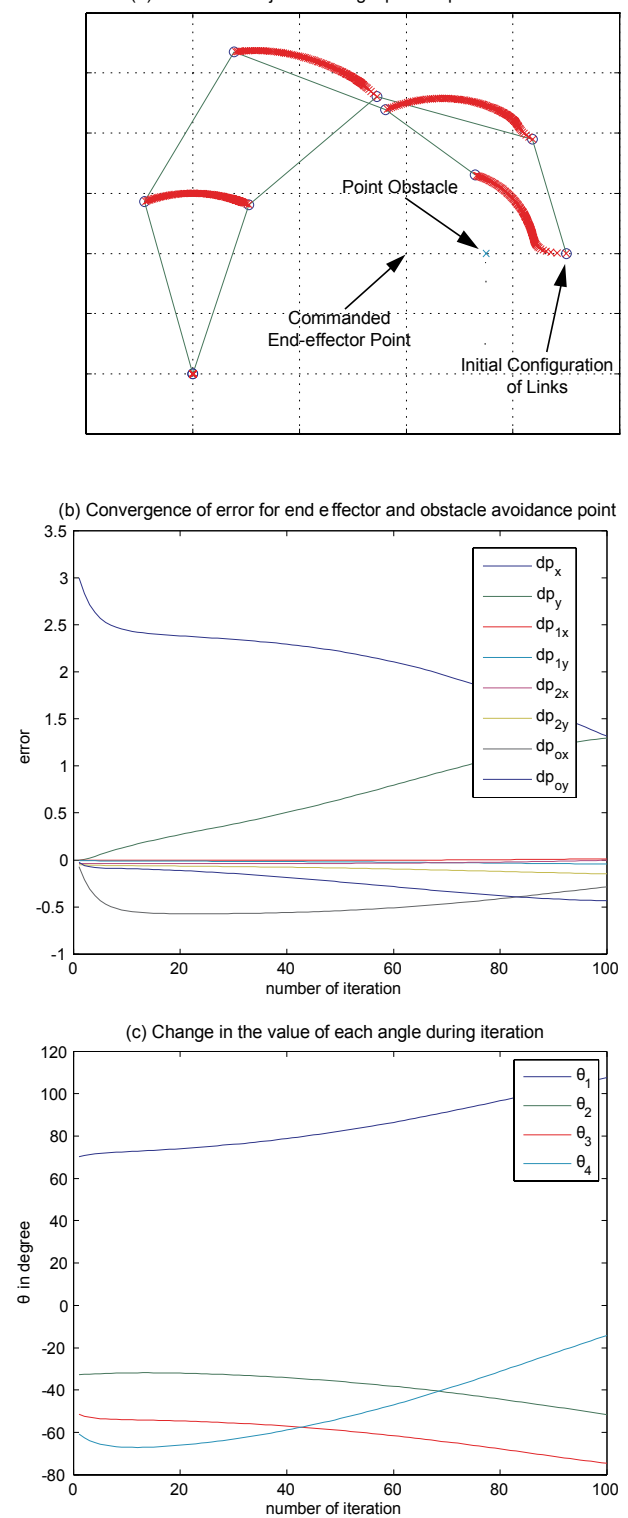

Figure 5 Simulation Results Without Applying Joint Directionality Scheme ( $\left.s_{1}=s_{2}=0, s_{o}=0.2\right)$

Lastly, by setting $s_{1}$ and $s_{2}$ to 0.01 , the model could reach the desired point without passing through obstacle region, and with desired path. Furthermore, the system was stabilized only after about 25 times of iteration. It was noticed in Fig. 6-(c) that small oscillation arises for each angle. The oscillation is due to the recursive characteristic of numerical method which is minimizing errors for every term simultaneously, which does not seem to affect much to the stability of system.

(a) Trace of each joint for single point to point command
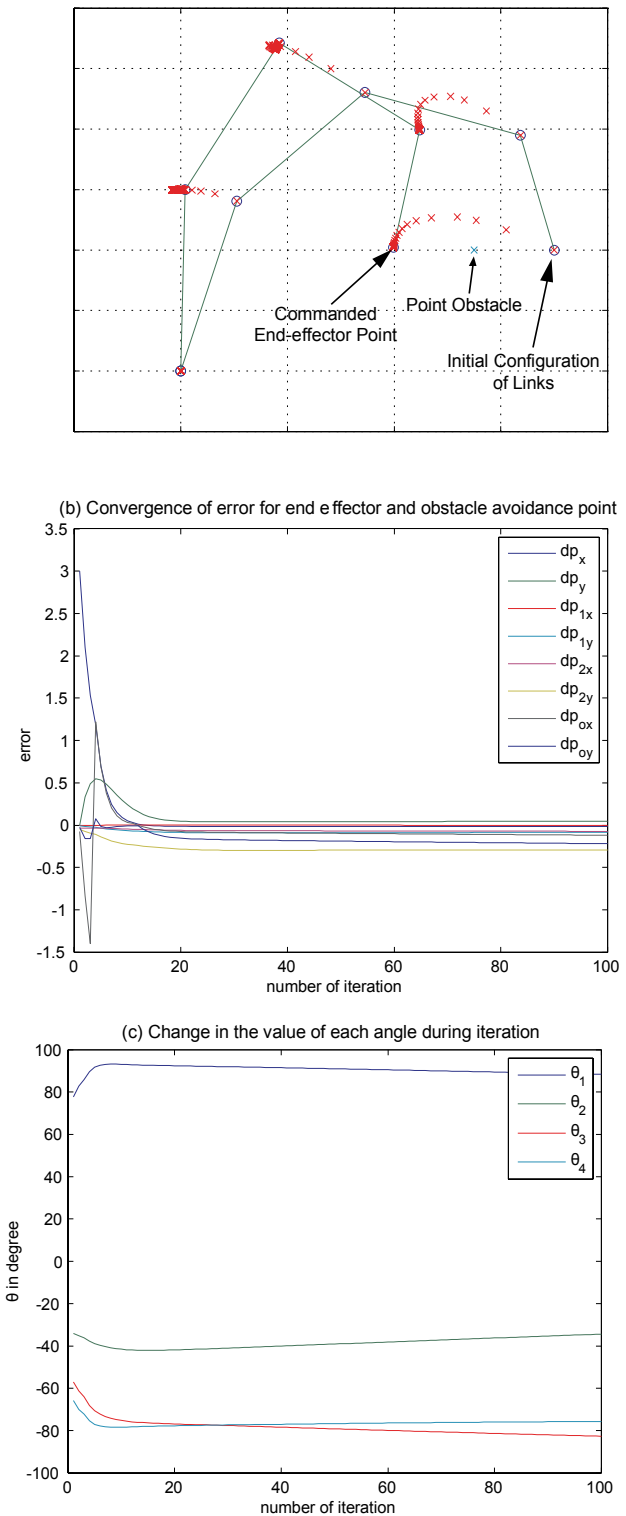

Figure 6 Simulation Results with Applying Joint Directionality Scheme ( $\left.s_{1}=s_{2}=0.01, s_{o}=0.2\right)$

\section{CONCLUSION}

Overall, as can be seen in the simulation results, joint directionality scheme has proven to be an effective approach for joint constraint and 
obstacle avoidance. In spite of the fact, further research is required to apply the scheme to real backhoe model. Real backhoe model can be thought of as a 4-link manipulator consisting of three links moving within a plane created by rotation of one link; swing motion, about $z$-axis. Therefore, there is discrepancy between real backhoe model and the one considered on this paper. Therefore, the discrepancy must be removed in further study. Besides, criterion for assigning adequate gain terms for each link, in equation (12), must be defined.

\section{ACKNOWLEDGMENT}

This research was supported by a grant (code\#06C01) from Unified and Advanced Construction Technology Program funded by Ministry of Construction \& Transportation of Korean government.

\section{REFERENCES}

[1] S. Singh, Synthesis of Tactical Plans For Robotic Excavation, Doctoral Dissertation, Tech. Report CMU-RI-TR-95-27, Robotics Institute, Carnegie Mellon University, January, 1995.

[2] F. Joseph George, Development of a Haptic Backhoe Testbed, The Degree of Master of Science in Mechanical Engineering, Georgia Tech Theses and Dissertations, May 2004.

[3] A. Stentz, J. Bares, S. Singh and P. Rowe, A Robotic Excavator for Autonomous Truck Loading, Autonomous Robots, Vol. 7, No. 2, pp. 175-186, September 1999.
[4] J. Denavit and R.S. Hartenberg, A Kinematic notation for lower-pair mechanisms based on matrices, ASME J. Apple, Mech., vol. 22, pp. 215-221, June 1955.

[5] C. R. Rao, S. K. Mitra, Generalized Inverse of Matrices and Its Applications, New York: Wiley, 1971

[6] T. Yoshikawa, Analysis and Control of Robot Manipulators with Redundancy, Robotics Research: The First International Symposium, pp. 735-747, MIT Press, 1984.

[7] H. Hanafusa, T. Yoshikawa, and Y. Nakamura, Analysis and control of articulated robot arms with redundancy, Prep. 8th IFAC World Congress. pp. XIV-78-83, August 1981.

[8] Y. Zhang and J. Wang, Obstacle avoidance for kinematically redundant manipulators using dual neural network, IEEE Transactions on Systems, Man, and Cybernetics - Part B: Cybernetics, vol. 34, no. 1, pp. 2747-2752, February 2004.

[9] A.A. Maciejewski and C. A. Klein, Obstacle avoidance for kinematically redundant manipulators in dynamically varying environments, Int. J. Robotics Res., vol. 4, no. 3, pp. 109-117, Fall 1985.

[10] L. Sciavicco and B. Siciliano, A Solution Algorithm to the Inverse Kinematic Problem for Redundant Manipulators, IEEE Journal of Robotics and Automation, vol. 4, no. 4, pp. 403-410, August 1988. 